Migräne

\title{
Was passiert im Gehirn bei einer Aura?
}

\begin{abstract}
— Sehstörungen während einer Migräneaura können unterschiedlicher Natur sein - von der Wahrnehmung von flimmernden Blitzen bis hin zu Gesichtsfeldausfällen. Die der Aura zugrunde liegenden Mechanismen sind allerdings aktuell nicht gut verstanden. Magdeburger Forscher haben nun mithilfe der funktionellen Kernspintomografie (fMRT) den neuronalen Mechanismen der visuellen Symptomen nachgespürt, um so auch Zugang zu neuen Therapieansätzen zu bekommen, heißt es in einer Pressemitteilung der Universität Magdeburg.

Wissenschaftler des Dänischen Kopfschmerzzentrums, der Neurologie der Universität Kopenhagen und der Universitätsaugenklinik Magdeburg haben herausgefunden, dass verschiedene Aura-Symptome unterschiedliche Funktionsveränderungen im Gehirn widerspiegeln. Mit fMRT untersuchten sie fünf Patienten während Aura-Attacken [Arngrim et al. Ann Neurol 2017;82(6):925-39].
\end{abstract}

Sobald die Patienten das Auftreten und
Fortschreiten von Sehstörungen berichteten, wurden sie sofort im Kernspintomografen untersucht. Dort wurden die Antworten der Sehrinde des Gehirns auf bewegte Schachbrettmuster gemessen.

Es zeigte sich, dass Patienten mit visuellen Aura-Symptomen wie Gesichtsfeldausfällen, erniedrigte Antworten der Sehrinde hatten, während Patienten, die beispielsweise Lichtblitze und Flimmern wahrnahmen, vergrößerte Antworten hatten, heißt es in der Universitätsmitteilung. Betrafen die visuellen Symptome beide Gesichtsfeldhälften, so wurden die Änderungen der Aktivität der Sehrinde auch in beiden Hirnhälften beobachtet.

Somit zeigten die Arbeitsgruppen dieses Kooperationsprojektes, dass unterschiedliche Aura-Symptome mit unterschiedlichen Antworten der Sehrinde zusammenhängen. Das verbesserte Verständnis der MigräneAura könnte auch Konsequenzen für eine effektive Behandlung haben, hoffen die Forscher.

(run)

\section{Ausschreibung}

\section{Förderpreis für ambulante Palliativversorgung}

— Die Deutsche Gesellschaft für Palliativmedizin (DGP) verleiht in diesem Jahr zum elften Mal den mit $10.000 €$ dotierten "Anerkennungs- und Förderpreis für Ambulante Palliativversorgung". Stifter ist die Grünenthal GmbH. Die DGP ruft in einer Pressemitteilung dazu auf, Anträge für den Preis bis zum 31. März 2018 beim Präsidenten der DGP einzureichen.

Die Ausschreibung richtet sich an Personen, Gruppierungen oder Institutionen, die sich in besonderer Weise um die Qualitätsentwicklung der ambulanten Palliativversorgung verdient gemacht haben, heißt es in der Mitteilung. Die Bezeichnung Anerkennungs- und Förderpreis bedeutet, dass bereits geleistetes Engagement anerkannt und gleichzeitig eine zukünftige Weiterführung gefördert wird.

Die eingereichten Projekte sollten einen wesentlichen Beitrag zur ambulanten Palliativversorgung darstellen und über den eigenen Bereich hinaus wirken. Der Nachweis dazu muss durch Forschungsund Projektbeschreibungen, Fachgutachten oder entsprechende Publikationen erfolgen. Arbeiten können von allen in der Palliativmedizin tätigen Berufsgruppen eingereicht werden.

Die prämierten Arbeiten werden während des 12. Kongresses der DGP vom 5.-8. September 2018 in Bremen vorgestellt.

Anträge für den Preis gehen an den Präsidenten der Deutschen Gesellschaft für Palliativmedizin, Aachener Str. 5, 10713 Berlin, E-Mail: dgp@palliativmedizin.de.

Die Statuten des Förderpreises und weitere Informationen sind über die Website der DGP abrufbar.

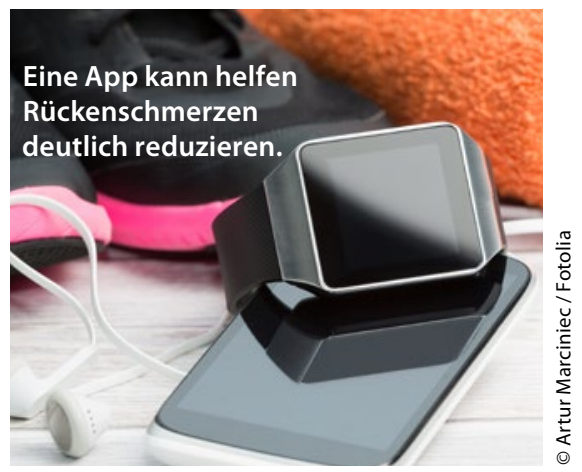

\section{Therapie per Smartphone \\ Per App gegen den Rückenschmerz}

— Schmerzen im unteren Rücken können durch eine Therapie per Smartphone verbessert werden, wie eine Studie ergeben hat [JMIR Rehabil Assist Technol 2017; 4(2):e11]. Untersucht wurden insgesamt 180 Patienten im Alter von durchschnittlich 34 Jahren, die zwölf Wochen lang die medizinisch geprüfte Rückentherapie-App Kaia nutzten.

Die Patienten durchliefen mit der App täglich ein 15- bis 30-minütiges Training, das sich mittels einer künstlichen Intelligenz stetig an die individuellen Bedürfnisse anpasste. Die Therapie umfasste dabei 150 Bewegungs- und Entspannungsübungen und die Vermittlung von Wissen über die Krankheit und ihre Therapie.

Die Forscher erfassten Nutzungsdauer und Einfluss auf das Schmerzlevel über eine numerische Skala - abhängig davon, ob die Rückenschmerzen akut, subakut oder chronisch waren. Durchschnittlich sanken die Schmerzen von 4,8 auf 3,8 Punkte, wie der Hersteller Kaia Health mitteilt. Bei Nutzern, die die App mehr als 12 Wochen nutzen, sanken die Werte von 4,6 auf bis zu 2,6 Punkte.

Kaia wurde kürzlich in einem Wettbewerb der besten Gesundheits-Apps während der Medizinmesse Medica mit dem dritten Platz ausgezeichnet. Kaia selbst ist ein Medizinprodukt der Klasse I und wurde vom TÜV Süd nach der MedizinprodukteherstellerNorm ISO 13485 zertifiziert.

Die App soll Patienten dazu motivieren, täglich zu Hause zu trainieren. Die Preise für die Nutzung von Kaia beginnen bei 79,99€ im Jahr.

(eb) 